\title{
The dynamics of merge and inversion of cavity and slot modes in coupled cavity chain slow wave structure
}

\author{
Irina Nakrap $^{1}$ and Alexander Savin ${ }^{2, *}$ \\ ${ }^{1}$ Saratov State University, Saratov, 410012, Russian Federation \\ 2JSC “RPC “Istok” named after Shokin”, Fryazino, 141190, Russian Federation
}

\begin{abstract}
The results of a theoretical study of the electrodynamic characteristics (EDC) in the dynamics of merge of the cavity and slot passbands of a slow-wave structure (SS) such as a coupled cavity chain (CCC) with the subsequent transition to a CCC with an inverted slot mode with a change in the opening angle of the coupling slot are presented. An analysis of the characteristics of CCC with combined and inverted bands with a traditional SS is carried out. The coupling impedance is presented for the first three spatial harmonics. The EDC CCC calculation was performed in the ANSYS HFSS program using the method of separation of closely spaced modes proposed by the authors. Suggested method made it possible to significantly increase the accuracy of the calculation of the dispersion characteristics of the $\mathrm{CCC}$ in the process of combining the passbands with the determination of the boundaries of not only the cavity, but also the slot passbands until the disappearance of the non-transmission band. The analysis of the distribution of the fields at given phase shifts in the cavity and slot CCC passbands revealed the features of their change during the inversion of the bands compared to traditional concepts. The field distributions in both passbands at different phase shifts have a hybrid (mixed) character of the fields of both the cavity and slot types waves.
\end{abstract}

\section{Introduction}

The coupled cavity chain (CCC) are widely used in high-power TWT. CCC with two sets of bandwidths: cavity and slot wave types, can be used in TWT in various operating modes. When the main cavity and slot passbands of CCC merged, a significant increase in its working passband was obtained in [1-3] (up to 70\% when the phase velocity changes within $\pm 3 \%$ ). The transition to the inverted slot mode slow wave structure (SS) significantly changes the properties of CCC and, accordingly, the functional application in TWT when suppressing excitation at the short-wavelength boundary of the working band $[4,5]$. In $[6,7]$, it was precisely the condition for the merge of the SS modes that was used in TWT when it was working in the main SS cavity band at phase shifts of $1.6 \pi \div 1.8 \pi$. This prevented oscillations at the border of the band, as well as increasing the bandwidth. In these works, filter-type SS were used: CCC in [6] and inductively loaded rectangular

* Corresponding author: savinan01@yandex.ru 
interdigital SS in [7]. But in [2,8], in-band instability at the backward wave in TWT at CCC was revealed in the region of band merging (at the point $\varphi=2 \pi$ ). Significant instability of TWT during bands merge in [2] required its suppression by nonreciprocal means. In [8], it is for CCC that the appearance of excitation at the boundary during modes combination is noted. Moreover, for the SS type of "ladder" structure with longitudinal communication channels (from the Millitron series), an important property is the low sensitivity to instability at the boundary of the working passband when it merges with the slot [8]. Thus, the study of the conditions and reasons for the possible instability of TWT on CCC when modes combined is an urgent task.

A complete study of electrodynamic characteristics (EDC) in the dynamics of the merge and inversion of the SS CCC cavity and slot passbands with their simultaneous expansion and dispersion characteristics (DC) control of both passbands with a wide variation of the main dimensions of the cavities was carried out in $[3,9,10]$. Optimization of CCC with combined passbands made it possible to obtain characteristics with a smooth change in their DC and a finite value of the coupled impedance in the merged region [3,9]. Nevertheless, the characteristics of the transmission coefficient and distribution of the SS field showed the presence in this region of a non-transmission band of small width (less than $2 \%$ ) with slight attenuation, as well as the excitation of a local oscillation in it [11]. Incomplete bands merge may be a condition for possible TWT instability in the combined mode. Thus, the TWT simulations on CCC during modes merge show the need for a significant increase in the accuracy of calculating the CCC DC with the determination of the boundaries of combined passbands, especially in the region of their steep dispersion.

This work is devoted to the theoretical study of EDC CCC in the dynamics of merge and inversion of its cavity and slot passbands in the transition from traditional CCC. The SS characteristics were calculated in the ANSYS HFSS program using the method of separation of closely spaced modes of resonator SS oscillations developed by the authors of this work. Particular attention is paid to the analysis of the distribution of fields at given phase shifts in the cavity and slot CCC passbands in order to detect changes in their character during merge and inversion of the passbands in comparison with the fields of traditional CCC.

\section{Object and methods of research}

The longitudinal and transverse sections of the SS type under study are a chain of inductively coupled cavities with $180^{\circ}$ bean-shaped coupling slots in adjacent cells and symmetrical drift tubes, shown in Fig. 1.

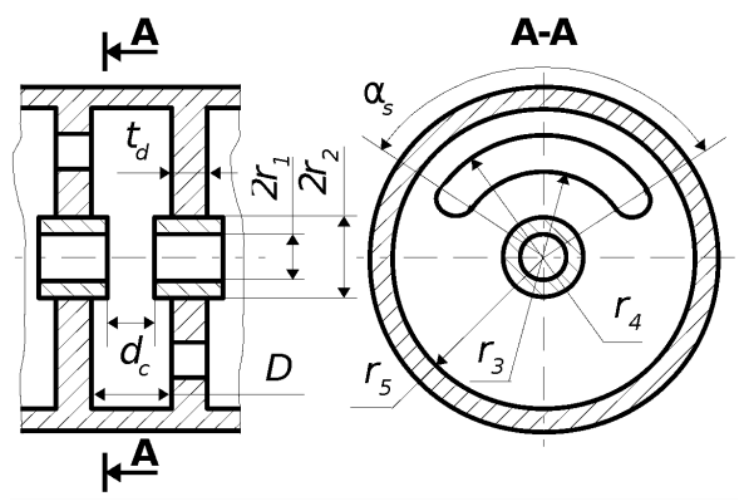

Fig. 1. Longitudinal and transverse sections of CCC. 
The CCC mockups designed to calculate the EDC in resonance mode are sections of Fig. 1. System with a finite number of cells short-circuited along the transverse planes of its symmetry by electric or magnetic walls. In the segments of the studied SS, the number of periods $\mathrm{N}$ was chosen equal to six $(\mathrm{N}=6)$.

Calculation in the ANSYS HFSS program using the finite element method of the resonant frequencies $f_{\mathrm{m}}$ of the SS segment with the number of periods $N$ and determination of the corresponding phase shifts $\varphi_{\mathrm{m}}=\pi m / N(m=0,1, \ldots, N) \varphi_{\mathrm{m}}$ using spectral analysis of the electric field distribution on the SS axis allows you to get $N+1$ point on DC SS for each studied passband. The results of calculating the resonant frequencies and the corresponding field distributions of the segments SS are also used to determine the coupling impedance $R_{\mathrm{c}}$ of spatial harmonics at the resonant frequencies $f_{\mathrm{m}} \mathrm{SS}$.

The dispersion characteristics of SS CCC in the cavity and slot passbands were calculated using the method of separation of closely spaced modes of resonator SS proposed in [12]. This made it possible to calculate with high accuracy the types of oscillations at phase shifts $\varphi=0$ in the region of incomplete merge, as well as in the case of inversion of the cavity and slot passbands.

\section{Results of research}

In contrast to our previous works [9], the combination of the CCC cavity and slot passbands, as well as their inversion (the case when the resonant wavelength of the slots becomes larger than the cavity's own wavelength), was carried out in the traditional way of simultaneously reducing the cavity diameter and increasing the opening angle of the coupling slot (see works $[1,2,4,5]$ ).

The relative sizes of SS CCC used in the study are shown in Table 1.

Table 1. Dimensions of SS CCC.

\begin{tabular}{|c|c|c|c|c|c|c|c|c|}
\hline № SS & $\boldsymbol{d}_{\mathbf{c}} / \boldsymbol{D}$ & $\boldsymbol{t}_{\mathbf{d}} / \mathbf{D}$ & $\mathbf{2} \boldsymbol{r}_{\mathbf{5}} / \boldsymbol{D}$ & $\boldsymbol{r}_{\mathbf{4}} / \mathbf{2} \boldsymbol{r}_{\mathbf{5}}$ & $\boldsymbol{r}_{\mathbf{3}} / \boldsymbol{r}_{\mathbf{4}}$ & $\boldsymbol{r}_{\mathbf{2}} / \boldsymbol{r}_{\mathbf{5}}$ & $\boldsymbol{r}_{\mathbf{1}} / \boldsymbol{r}_{\mathbf{2}}$ & $\boldsymbol{\alpha}_{\mathbf{s}}(\mathbf{g r a d})$ \\
\hline 1 & 0.335 & 0.129 & 2.284 & 0.5 & 0.678 & 0.311 & 0.755 & 100 \\
\hline 2 & 0.335 & 0.129 & 2.284 & 0.5 & 0.678 & 0.311 & 0.755 & 132.42 \\
\hline 3 & 0.335 & 0.129 & 2.284 & 0.5 & 0.678 & 0.311 & 0.755 & 190 \\
\hline
\end{tabular}

The dispersion characteristics of the working spatial harmonics and the coupling impedance of the first three spatial harmonics SS CCC in the dynamics of combination (SS No 2) and inversion (SS No 3) of the cavity and slot passbands with the transition from the traditional CCC (SS No. 1) are shown in Fig. 2, 3. SS No 2 with combined cavity and slot passbands has a working band of $60 \%$ with a change in phase velocity within $\pm 3 \%$ (Fig. 2). Its coupling impedance in this band for working spatial harmonics ("- 1 " in the cavity and " +1 " in the slot passbands) varies from $20 \mathrm{Ohms}$ to $0.5 \mathrm{Ohms}$ (Fig. 3b). The coupling impedances have approximately the same values for the corresponding harmonics of the cavity and slot passbands. Moreover, when approaching the boundary $(\varphi=2 \pi)$, the coupling impedance "-1" of the spatial harmonic does not tend to infinity (Fig. 3b) like the traditional SS No 1 (Fig. 3a) and remains finite, and the coupling impedance is " +1 " spatial harmonics does not tend to zero and also remains finite.

The coupling impedance of the first three spatial harmonics SS No 3 (Fig. 3c) in the inverted slot band becomes higher than the coupling impedance of the corresponding harmonics of the cavity passband. In this case, when approaching the boundary $(\varphi=2 \pi)$, the coupling impedance of the working spatial harmonics ("- 1 " in the cavity passband and " +1 " 
in the slot passband) tend to zero. This behavior of the coupling impedance of the working harmonics of the SS CCC with the inverted slot mode allows one to avoid self-excitation at the short-wavelength boundary of the working band when used in TWT.

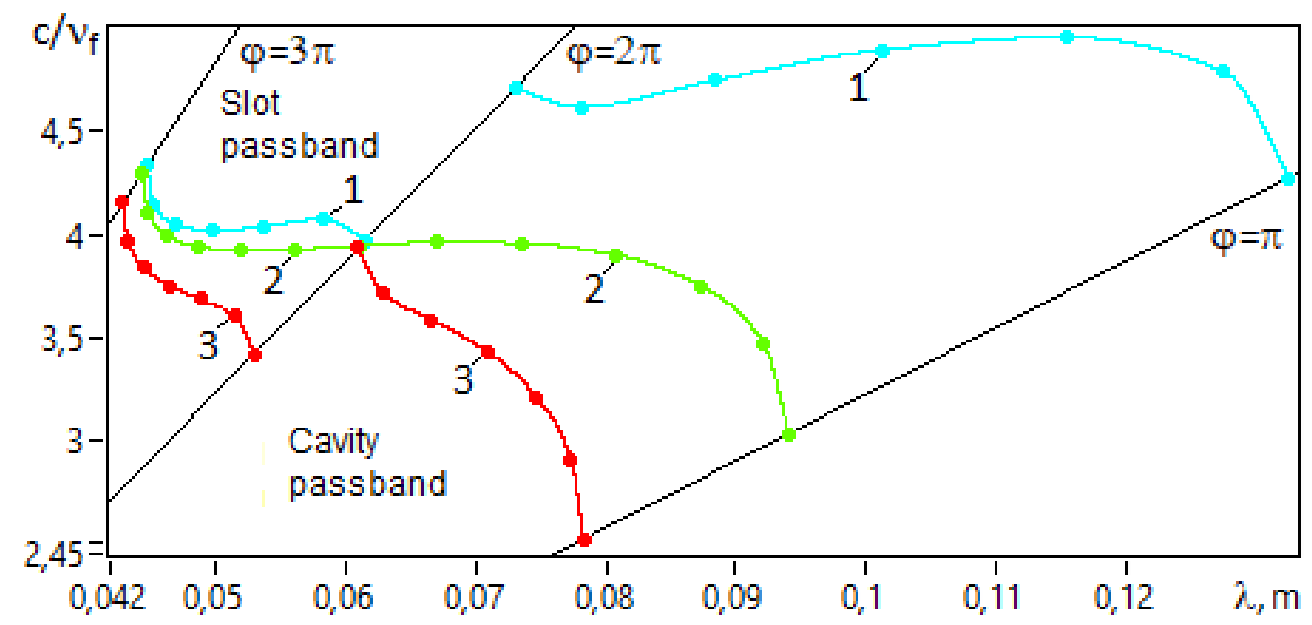

Fig. 2. The dynamics of merge of the cavity and slot passbands SS CCC depending on the angle of the opening of the coupling slot $\alpha_{\mathrm{s}}$ with constant period $D .1-\alpha_{\mathrm{s}}=100^{\circ}, 2-\alpha_{\mathrm{s}}=132.42^{\circ}, 3-\alpha_{\mathrm{s}}=190^{\circ}$.

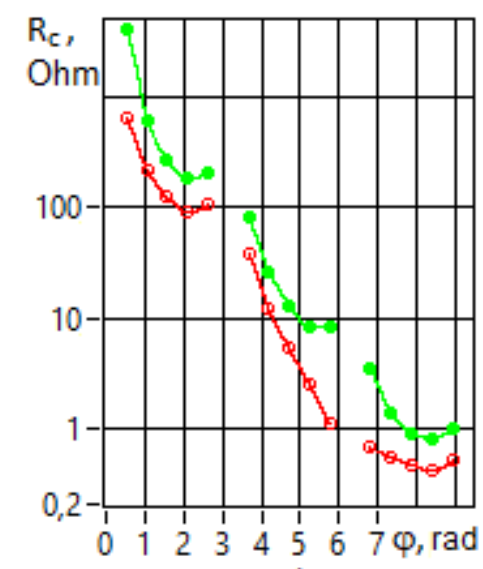

a)

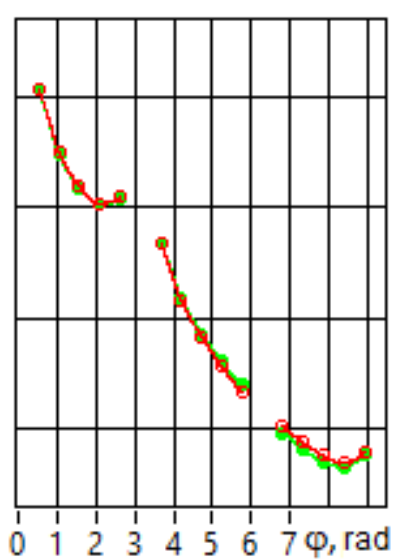

b)

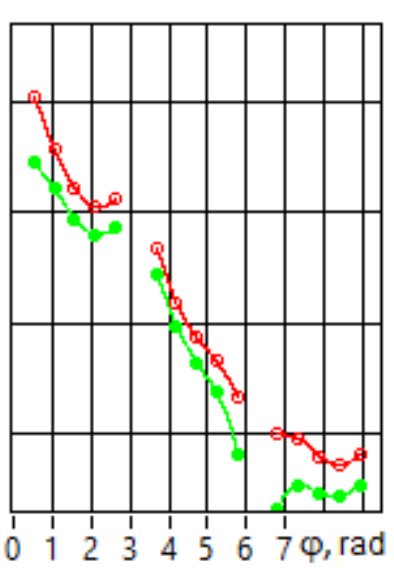

c)

Fig. 3. The coupling impedance is " 0 ", " +1 ", " -1 " of the spatial harmonics of the cavity $(\square)$ and slot (O) passbands SS CCC depending on the angle of the opening of the coupling slot $\alpha_{\mathrm{s}}$ with constant period $D$. a) $-\alpha_{\mathrm{s}}=100^{\circ}$, b) $\left.-\alpha_{\mathrm{s}}=132.42^{\circ}, \mathrm{c}\right)-\alpha_{\mathrm{s}}=190^{\circ}$.

The electric field distributions for all calculated resonant modes of oscillation in the cavity and slot passbands for SS No 1-3 are shown in Fig. 4, 5.

In all three SS (Fig. 4, 5) in the low-frequency and high-frequency passbands, the field distribution at large phase shifts $(\varphi=\pi 4 / 6, \ldots, \pi 6 / 6)$ retains the character of the traditional CCC (SS No 1). At the phase shifts $\varphi=0$ in SS No 3, the resonant wavelength of the slots becomes greater than the cavity's own wavelength and the field distributions in the lowfrequency and high-frequency passbands are swapped compared to the traditional CCC.

At small phase shifts $(\varphi=\pi 1 / 6, \ldots, \pi 3 / 6)$ in SS No 3, the field distributions in both passbands have both resonator and slot type components, i.e. the field is hybrid compared to traditional CCC. 


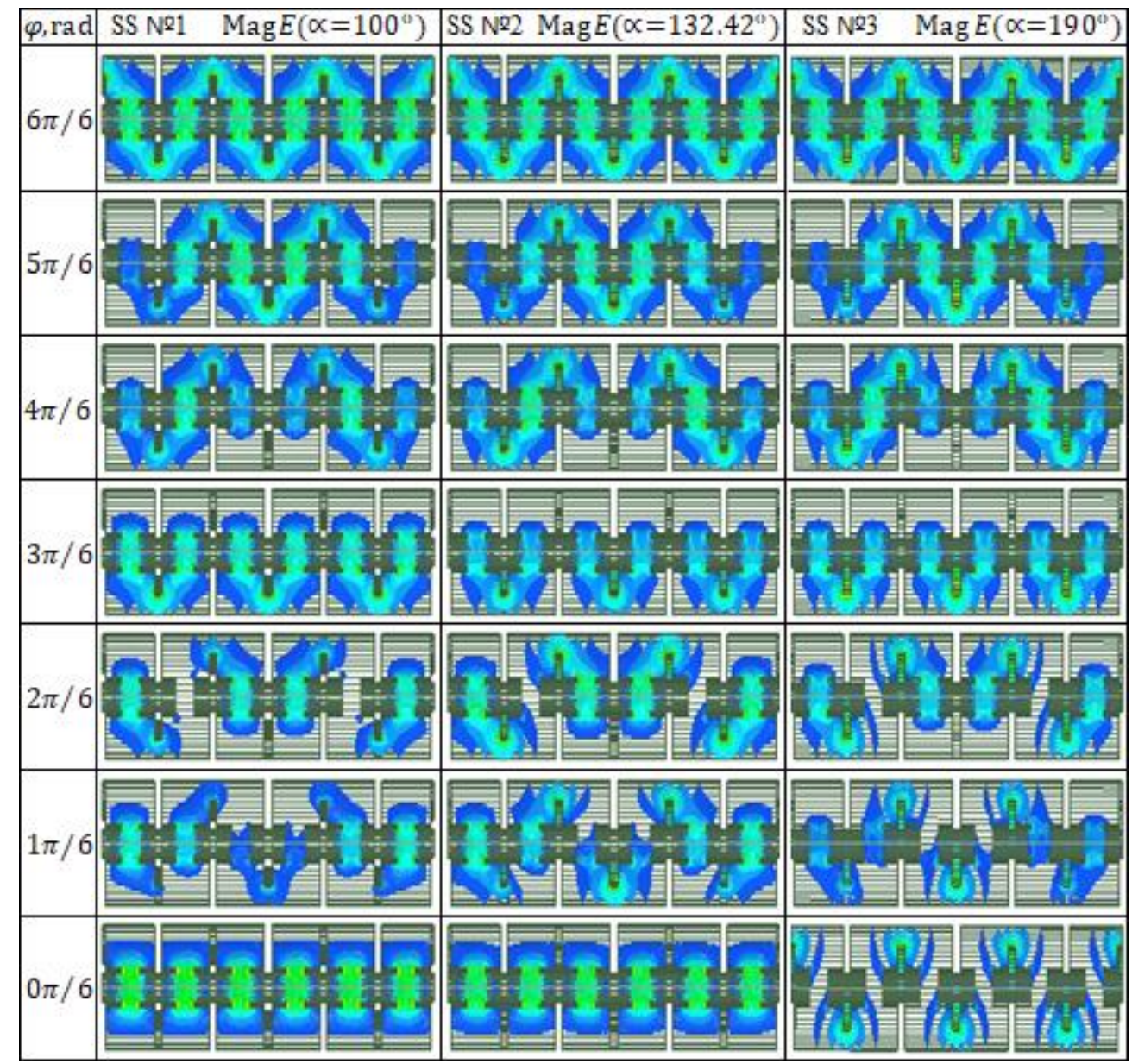

Fig. 4. The distribution of the electric field on the resonant modes of oscillation in the low-frequency passband SS CCC.

Thus, when the passbands are inverted in SS No 3, the cavity passband at small phase shifts obtains properties of the slot passband, which explains the behavior of the coupling impedance of the spatial harmonics of the SS CCC with the inverted slot mode (Fig. 3c) compared to the traditional SS CCC (Fig. 3a).

\section{Conclusion}

A study was made of the dynamics of the merge of the cavity and slot passbands of SS CCC and the subsequent transition to their inversion. The finite element method has been used to calculation the electrodynamic characteristics of SS CCC, including the distribution of fields in the main (cavity) and higher (slot) passbands, including at the boundaries of the bands.

The analysis of the distribution of fields at given phase shifts in the cavity and slot passbands of CCC revealed the peculiarities of their changes during the inversion of the bands compared to traditional concepts. It was revealed that the field distributions in both bands at small phase shifts have a hybrid (mixed) character of the fields of both the cavity and slot types waves, which explains the behavior of the coupling impedance of the working spatial harmonics of the SS CCC field. 


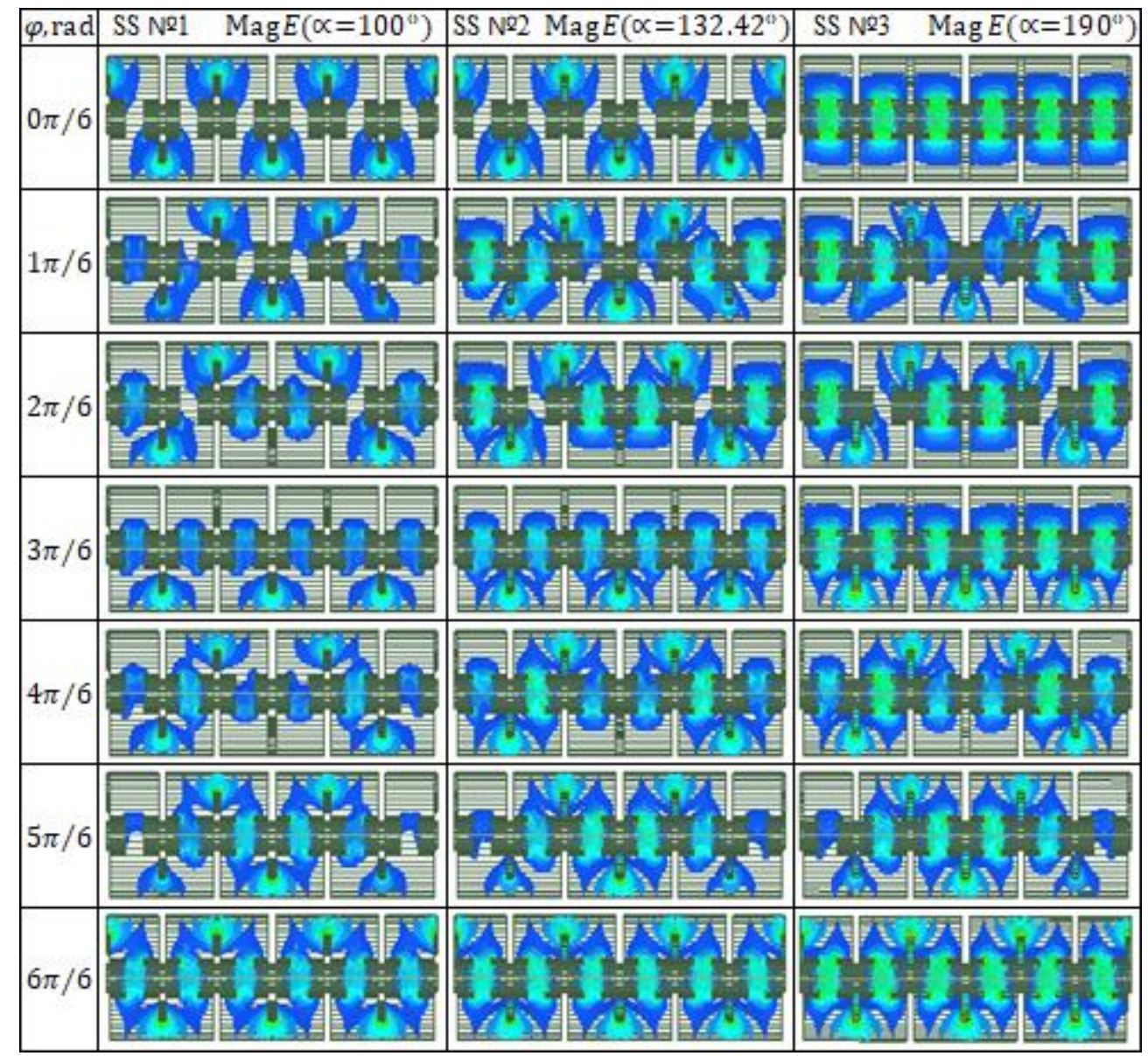

Fig. 5. The distribution of the electric field in resonance modes in the high-frequency passband SS CCC.

\section{References}

1. A. Karp, W.R. Ayers Design concepts for an octave-band width coupled-cavity TWT/ IDEM, Washington, pp. 546-549 (1978)

2. O. Sauseng, M.E. Triplett A $5 \mathrm{~kW}$ wideband coupled cavity tube with ppm focusing for $11 \mathrm{GHz}$ to $17 \mathrm{GHz} / \mathrm{IDEM}$, Washington, pp. 491-493 (1974)

3. I.A. Nakrap, A.N. Savin, Yu.P. Sharaevskii Modeling of Wideband Slow-Wave Structures of Coupled-Cavities-Chain Type by the Impedance Design of Experiments. Journal of Communications Technology and Electronics. 51 (3), pp. 333-340 (2006)

4. J.R. Frey, I. Tammaru A coupled cavity TWT operating in the inverted slot mode / IDEM, Washington, pp. 504-506 (1981)

5. L. Kumar, V.L. Christie, Balakrishnan N. Inverted slot mode slow wave structures for traveling wave tubes. IEEE Transactions on Microwave Theory and Techniques. Vol. 55. № 6. pp. 1112-1116 (2007)

6. J.B. Kennedy, I. Tammaru and P.S. Wolcott Study of 42 AND 85 GHz Coupled Cavity TWTs for Space Use, NASA-134670 (1977) 
7. S. Nej, L. Christie Coupled resonator slow wave structures for millimetric wave TWT. International Conference on Energy, Communication, Data Analytics and Soft Computing, ICECDS (2017); Chennai; India. 19 June, pp. 1209-1211 (2018)

8. B.G. James et al. New Circuit for Generating High Power at Millimeter Wavelengths, MOGA 70, Amsterdam, Holland. pp. 5.7-5.13

9. I.A. Nakrap, A.N. Savin Electrodynamics characteristics of coupled-cavity chain with the inverted slot mode. Proceedings of the $27^{\text {th }}$ International Conference «Microwave \& Telecommunication Technology» (CriMiCo'2017) Sevastopol, Russia, September 10-16, pp. 199-207 (2017)

10. I.A. Nakrap, A.N. Savin Analiz vozmozhnosti ispol'zovaniya rezonatornogo i shchelevogo tipov voln v LBV na CSR s obrashchennoj shchelevoj modoj. // Problemy SVCH elektroniki. T. 3. № 1. S. 51-52 (2017)

11. I.A. Nakrap, A.N. Savin Characteristics of CCC in area of merging of cavity and slot passbands. Radiotekhnika, Vol. 83, No 7(9), pp. 13-24 (2019)

12. A.N. Savin, I.A. Nakrap, K.P. Vakhlaeva Method of separation of oscillations types in the calculation of characteristics of SWS CCC in the program ANSYS HFSS. Ural Radio Engineering Journal, Vol. 2, No.4. pp. 41-51 (2018) 